\title{
Collaborations around caries - in order to improve oral health, healthcare and health
}

\author{
Nigel Pitts, Guest Editor BDJ Caries Focus Issue and Professor of Dental Health; Honorary Consultant, Dental Public Health; Director of Innovation \\ and Impact; Faculty of Dentistry, Oral \& Craniofacial Sciences, King's College London, Guy's Dental Hospital, London, SE1 9RT, UK.
}

The BDJ Upfront section includes editorials, letters, news, book reviews and interviews. Please direct your correspondence to the News Editor,

Kate Quinlan at k.quinlan@nature.com. Press releases or articles may be edited, and should include a colour photograph if possible.

$\mathrm{I}$ am delighted to be able to bring together in this focused issue a range of diverse collaborations around caries and share eight stories from colleagues and collaborators around the world all making progress to improve oral health, healthcare and health. I must start by fully acknowledging the many individuals, groups, organisations and supporters who have made these long-term collaborations possible and sustainable. They continue to be inspiring and go over and above' personal, institutional and corporate commitments to make a difference. We should also remain conscious of the need to link and integrate the so-called 'downstream', 'midstream' and 'upstream' elements of the caries landscape.

Caries is still ubiquitous yet far too often ignored in clinical, public and policy debates. I hope that the information provided here can go some way to correcting that. The first paper outlines collaborations to make a difference in the caries space and also build wider impacts. It explores the range and complexity of enduring, long-term, typically voluntary collaborations needed to make beneficial change and looks at some of the characteristics and challenges of success. To create enduring impact, these collaborations must extend beyond academia and work 'glocally', using global evidence adapted for local circumstances.

Coping with new insights from science, as evidence shifts, and working through the clinical and public health implications is a challenge. The caries example in the second paper concerns changes in the science around cariology and microbiology, shifting the understanding of caries as a noncommunicable disease and the consequences of this. It is often hard but essential to shift both opinion leaders and policymakers from embedded but outdated concepts. As we now know that caries is not caused solely by one micro-organism (S. mutans), we are no longer trying to sterilise the oral cavity but focus on maintaining a healthy oral microbiome.

In the policy arena, we need to break out of our silos and talk to broader stakeholder groups. The 'win 6 cube' stakeholder approach developed at the three 'Dental Policy Labs' (DPLs) described in this issue has been very useful. DPL1 looked at how to accelerate progress 'towards a cavity-free future'; DPL2 looked 'towards paying for health in dentistry', developing a blueprint for doing so; while DPL3 'towards oral and dental health through partnership' explored behaviour change at public and patient levels as well as industry repeat restorative care to preventive dental medicine. It's gratifying to see peer recognition of both the quality of the published research, as well as the international implementation.

Innovation and technology have the potential to help us improve health and healthcare for our patients, but the process is long, complicated and requires very different groups to work together. The seventh paper looks at collaborations to improve dental caries activity assessment in clinical practice by providing new tools to address a longstanding unmet clinical need. It outlines the story from the first 'lightbulb' moment, to exploring the idea, raising finance and iterative research and

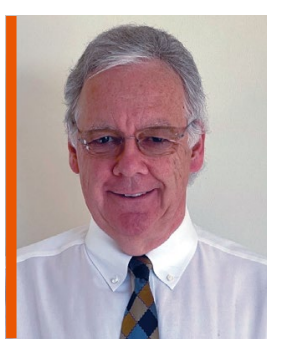

\section{'I think that there are now significant opportunities to [...] make real and} necessary progress towards a cavity-free future.'

partnerships. The reality that caries care has to be managed considering both professional and business perspectives was a recurring theme at these Labs, as was the need to look more broadly at the value of health. These Labs led to the development of a Making Cavities History Taskforce, ${ }^{1}$ which has produced a global consensus for policies to achieve a dental cavity-free future which fit well with the new WHO Oral Health Resolution, passed by the World Health Assembly in May, as well as with the FDI World Dental Federation's recent Vision 2030 strategy.

In the clinical arena, the sixth paper looks at the journey taken by long-term international collaborations to identify and secure optimal detection, assessment and management of caries in clinical practice, and the shift from development to securing regulatory approval for a novel technology.

Lastly, it is a pleasure to share details of the initial plans of the new $29^{\text {th }}$ Chapter of the Alliance for a Cavity-Free Future just established for the UK, which I co-Chair with Professor Avijit Banerjee. Putting all this together, I think that there are now significant opportunities to build on the work of all the collaborations outlined in this issue and make real and necessary progress towards a cavity-free future.

\section{Reference \\ 1. ACFF. Making Cavities History. 2021. Available at www. acffglobal.org/making-cavities-history/ (accessed October 2021).}

https://doi.org/10.1038/s41415-021-3615-6 